\title{
A SOLID-STATE ATOMIC FREQUENCY STANDARD
}

\author{
Thesis by \\ Christopher J. White \\ In Partial Fulfillment of the Requirements for the \\ degree of \\ Doctor of Philosophy
}

\section{CALIFORNIA INSTITUTE OF TECHNOLOGY}

Pasadena, California

2005

(Defended May $16^{\text {th }}, 2005$ ) 
(C) 2005

Christopher J. White

All Rights Reserved 


\section{ACKNOWLEDGEMENTS}

The author thanks Ali Hajimiri for his continuous support and guidance during the course of this project. Richard Bramley was very generous in discussing zero-field electron magnetic resonance and sharing details of his earlier spectrometer designs. James White oversaw the machining of the loop-gap resonator pieces at MIT Central Machine Shop. Thanks to Philip Feng from Roukes Group at Caltech for annealing the magnesium oxide crystals. Thanks also to William Bridges, David Rutledge, Daniel Weitekamp, Sander Weinreb, Yu-Chong Tai, and Michael Roukes for serving on the thesis committee and offering many helpful criticisms. The author acknowledges the support of an Intel Fellowship during one year of the project.

Donhee Ham was a good friend during my years at graduate school, and I should also mention the help and encouragement I received from my brother James in completing the dissertation. Thanks always to my wife Rachel for her love and support, to Olga and Tony for their hospitality, and to my parents Gerald and Sharlene. 


\begin{abstract}
The thesis describes a new class of frequency reference. The frequency source uses the same operating principle as a passive atomic frequency standard; however, the device is entirely solid-state, removing many cost and reliability issues associated with gas-phase atomic clocks. More specifically, the "atomic resonance" is derived from zero-field magnetic resonance transitions of the vanadium ion in a cubic crystal lattice. The characteristics of these resonances will be described in detail. The apparatus for measuring the "atomic" resonances uses a radio-frequency resonant cavity and frequency discriminator circuit. Using integrated circuits, the radio-frequency signal processing functions can be implemented at very low cost in a reliable manufacturing process. We discuss the system design and the measurement sensitivity. Advantages of the new frequency reference may include immunity to vibration and reduced aging compared to crystal oscillators.
\end{abstract}




\section{TABLE OF CONTENTS}

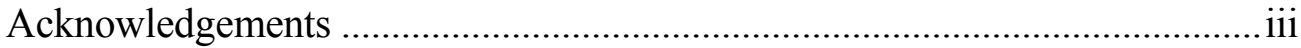

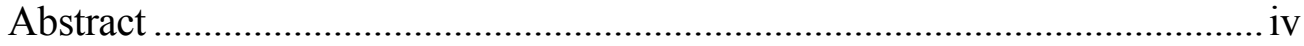

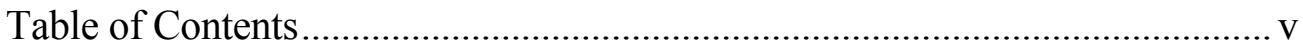

List of Figures ......................................................................................... vii

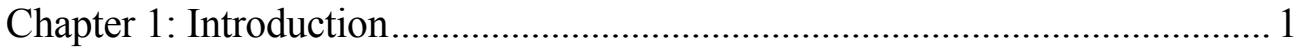

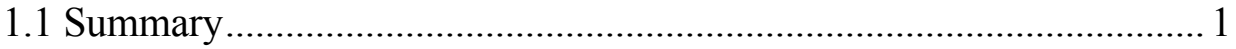

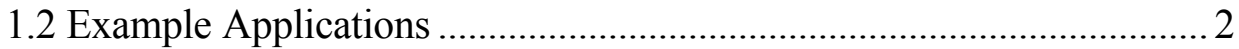

1.3 High-Accuracy, Low-Power Frequency Standards.............................. 4

1.4 Precision Crystal Oscillators .............................................................. 6

1.5 Miniaturized Rubidium Frequency Standards................................... 7

1.6 Solid-State Atomic Frequency Standard ........................................... 8

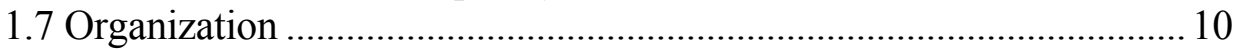

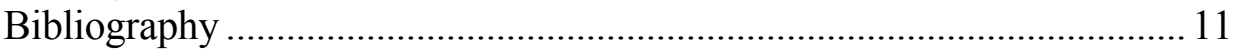

Chapter 2: Paramagnetic Resonance Signals ................................................ 14

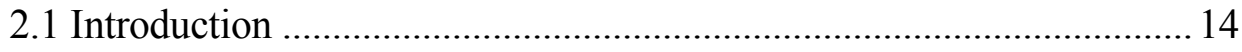

2.2 Transition Metal Paramagnetic Resonance in Solids ......................... 15

2.3 Zero-Field Electron Spin Resonance of Vanadium in $\mathrm{MgO}$................. 16

2.4 Hamiltonian for $\mathrm{V}^{++}$in a Cubic Lattice .............................................. 17

2.5 Magnetic Resonance at Zero-Field .................................................... 18

2.6 Selection Rules for Parallel and Perpendicular Polarizations .............. 23

2.7 Magnetic Susceptibility .................................................................... 25

2.8 Electrical Resonator Containing Paramagnetic Sample......................2 27

2.9 Numerical Calculation of Eigenvalues and Transition Intensities....... 29

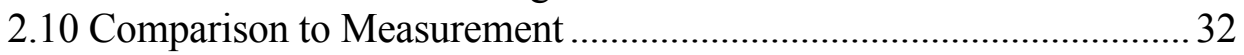

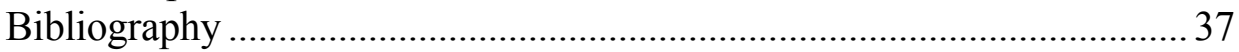

Chapter 3: Dipolar Broadening at Zero-Field ................................................ 39

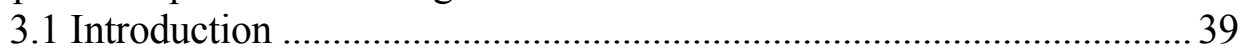

3.2 Dipolar Broadening of Dilute Paramagnetic Ions at High-Field ......... 40

3.3 Dipolar Broadening at Zero-Field....................................................... 42

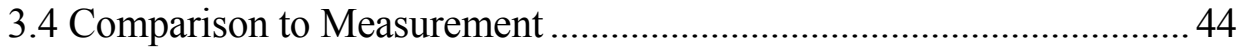

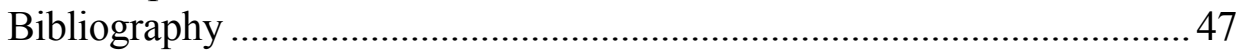

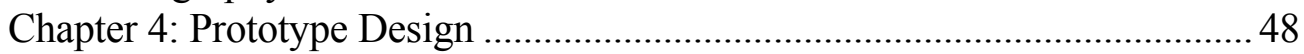

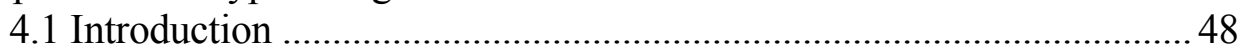

4.2 Tunable Loop-Gap Resonator Design ............................................... 52

4.3 Loop-Gap Resonator Manufacturing ……...........................................5 55

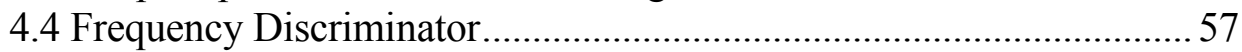

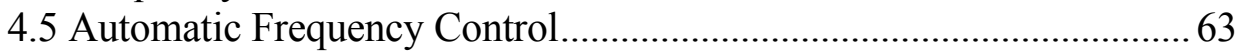

4.6 Modulation Waveforms and Modulation Driver...................................... 64

4.7 Helmholtz Coil Design and Magnetic Field Homogeneity.................. 65

4.8 Voltage-Controlled Oscillator and Phase Noise.................................. 67 


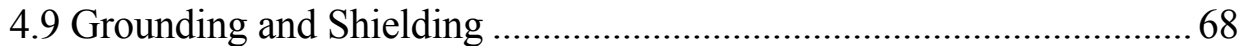

4.10 Sample Preparation and Heat Treatment ............................................6 68

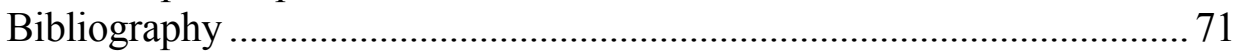

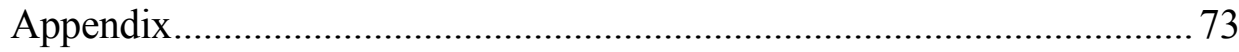

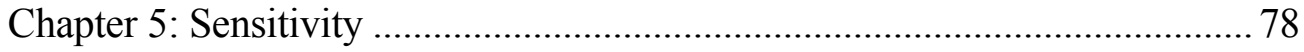

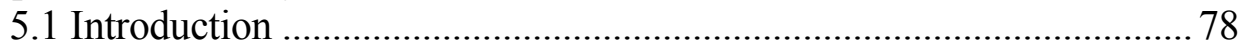

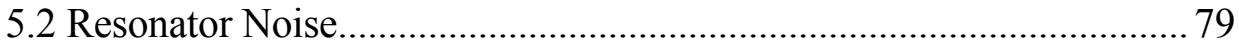

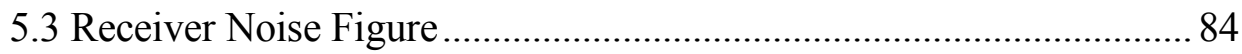

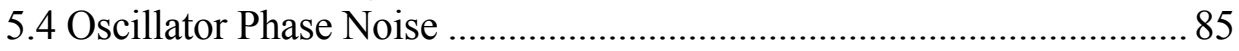

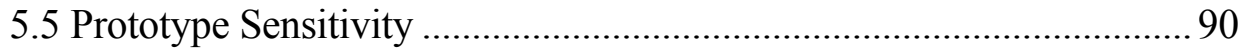

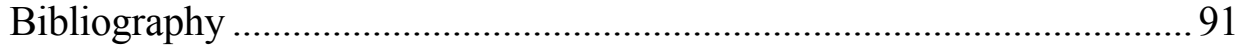

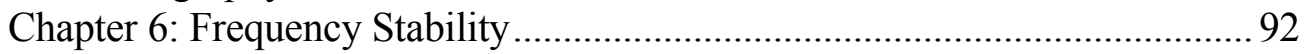

6.1 Frequency Stability of Passive Atomic Frequency Standards ............ 92

6.2 Measurement of Frequency Stability ................................................. 97

6.3 Solid-State Atomic Frequency Standard …...................................... 100

6.4 Frequency Stability - Thermal Noise Limit ....................................... 102

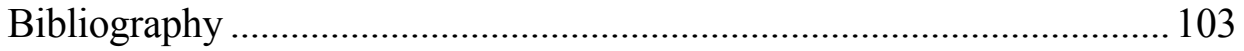

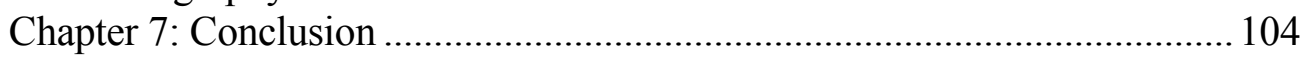




\section{LIST OF FIGURES}

Number Page

1.1. SARSAT Emergency Radiolocation Beacon ................................................ 3

1.2. Historical Trends in the Performance of Frequency Standards ........................... 5

2.1 Numerically Calculated Zeeman Shift for $\mathrm{V}^{++} / \mathrm{MgO}$.......................................22

2.2 Transition Intensities for Parallel and Perpendicular Polarizations ......................24

2.3 Calculated Spectrum for $\mathrm{Mn}^{++}$in Magnesium Oxide....................................... 30

2.4 Example Calculated Spectrum for $\mathrm{Mn}^{++}$and $\mathrm{V}^{++}$in Magnesium Oxide ............... 31

2.5 Measured Spectrum for $\mathrm{Mn}^{++}$and $\mathrm{V}^{++}$in Magnesium Oxide........................ 32

3.1 Numerically Calculated $\mathrm{V}^{++} / \mathrm{MgO}$ Spectrum Showing Zeeman Shift at 1 Gauss .... 42

3.2 Measured $\mathrm{V}^{++} / \mathrm{MgO}$ Spectrum at 50 Gauss Zeeman Field ................................ 45

4.1 Zero-Field Electron Magnetic Resonance Spectrometer....................................49

4.2 Shielded Loop-Gap Resonator: Perspective, Side and Top View ........................50

4.3 Audio Modulation Waveform and Lock-In Multiplier Waveform........................ 51

4.4 Mechanical Dimensions of Loop-Gap Resonator............................................ 53

4.5 Side View of Mechanically Tunable Loop-Gap Resonator ................................54

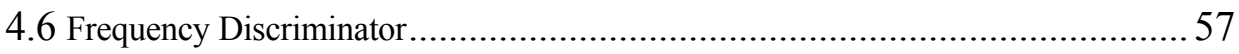

4.7 Power Variations at Mixer Input Because of Loop-Gap Resonator Mismatch........ 58

4.8 Measured Loop-Gap Resonator Transmission at 3 Frequencies..........................59

4.9 Measured Loop-Gap Resonator Reflection at 3 Frequencies ...............................6 60

4.10 Voltage Standing Wave Ratio without Series Matching Capacitor.................... 61

4.11 Power Variations at Mixer Input Port with Improved Resonator Matching.......... 62

4.12 Frequency Discriminator with Automatic Frequency Control .........................63

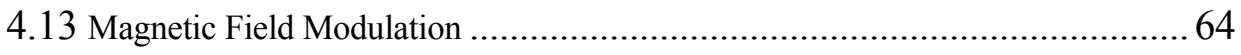

4.14 Helmholtz Coil Axial Magnetic Field Variation ............................................ 66

4.15 Measured Voltage-Controlled Oscillator Phase Noise ................................... 67

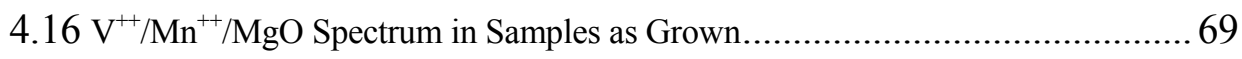

4.17 Spectrum after annealing 6 hours @ $1100^{\circ} \mathrm{C}_{\text {in }} \mathrm{H}^{2} /$ Argon Atmosphere .............. 70 


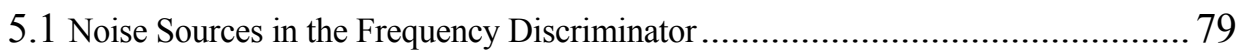

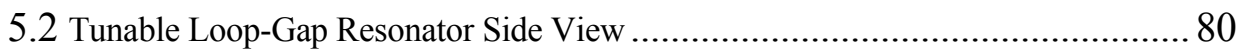

5.3 Lorentz Force Between Helmholtz Coils..................................................... 83

5.4 Background Pickup Signal During Spectrometer Sweep ................................. 84

6.1 Passive Atomic Frequency Standard .......................................................... 92

6.2 Atomic Resonance Signal Showing Instantaneous and Mean Frequency .............. 96

6.3 Direct Frequency Counter Measurement of Allan Variance............................... 97

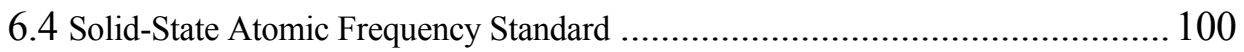

6.5 Frequency-Locking of Solid-State Atomic Frequency Standard ....................... 101 
Chapter 1

\section{INTRODUCTION}

\subsection{Summary}

Frequency standards are important in nearly all electronic systems, and are widely used in cellular telephones, wireless networks, satellite broadcasting, fiber-optic communication, global positioning systems (GPS), and a variety of instrumentation applications such as frequency synthesizers. Two classes of frequency standards exist; crystal oscillators and atomic clocks, which differ widely on their accuracy, size, cost, and power requirements [1]. Historically, the mainstream applications of crystal oscillators and atomic clocks have been very different. Most atomics clocks are used in laboratories for making highly precise measurements, or maintaining the basic standard of time. On the other hand, crystal oscillators are ubiquitous in consumer electronics, may cost only a few cents, are very small, and very low-power.

There is currently a need for low-cost, high-performance frequency standards which the solid-state atomic frequency standard presented in this dissertation seeks to address. Two technologies which are currently used for high-accuracy frequency control outside a laboratory environment are oven-controlled crystal oscillators and rubidium atomic clocks. However, the cost and power consumption of both technologies stands in the way of more widespread application of precision frequency control. Recent trends in frequency standards have been to aggressively shrink the size of rubidium atomic clocks and attempt to develop batch manufacturing techniques to lower the production cost $[2,3,4,5,6,7,8,9,10,11,12]$. For crystal oscillators, every possible refinement has been applied in the last few decades to improve the long-term accuracy and temperature stability $[13,14,15,16,17,18]$, and to reduce the power consumption of oven-controlled devices 
$[19,20,21,22,23]$. New solutions are needed to realize low-cost, portable frequency sources with improved accuracy.

\subsection{Example Applications}

Many developments in frequency standards have been motivated by the requirements of new communications systems or better navigation, which is likewise true of efforts to develop robust, miniature, low-power frequency standards which also have high-accuracy [24]. Two systems which illustrate the needs for improved frequency control are military spread-spectrum communications and commercial emergency radiolocation beacons $[25,26]$. The spread-spectrum example describes the need for a frequency reference with high-accuracy and low-vibration sensitivity. The radiolocation beacon example demonstrates the need for a frequency reference with good stability over temperature, lowpower consumption, and low-cost.

\section{Spread Spectrum Communications}

In the first case, the frequency accuracy requirements arise because of the narrow channel spacing in spread-spectrum communications systems. Channel spacing tends to be reduced to allow more users to operate in the same radio band and to make communications more difficult to intercept. In practical cases, channel bandwidths are often only a few $\mathrm{kHz}$, and with carrier frequencies extending up to X-band, frequency accuracy from 5-ppm to 0.001 ppm is required [25]. The frequency accuracy specification should be maintained under acceleration, and for a year or more without re-calibration. Also, phase noise caused by vibration sensitivity of the frequency reference is a limiting factor for the system capacity in some cases [25]. Vibration sensitivity is all the more critical for microwave systems, because audio frequency vibrations are multiplied to phase noise in adjacent channels. Therefore, for spread-spectrum communications, a frequency reference which has highaccuracy and low-vibration sensitivity is essential. 
Emergency Radiolocation

A second example related to navigation is a type of Doppler radiolocation system. Emergency radiolocation beacons allow a user to relay an emergency distress signal via satellite to a search and rescue ground terminal. The location of the distress beacon is detected by tracking the Doppler shift of the beacon signal seen by the satellite flying overhead (Figure 1.1):

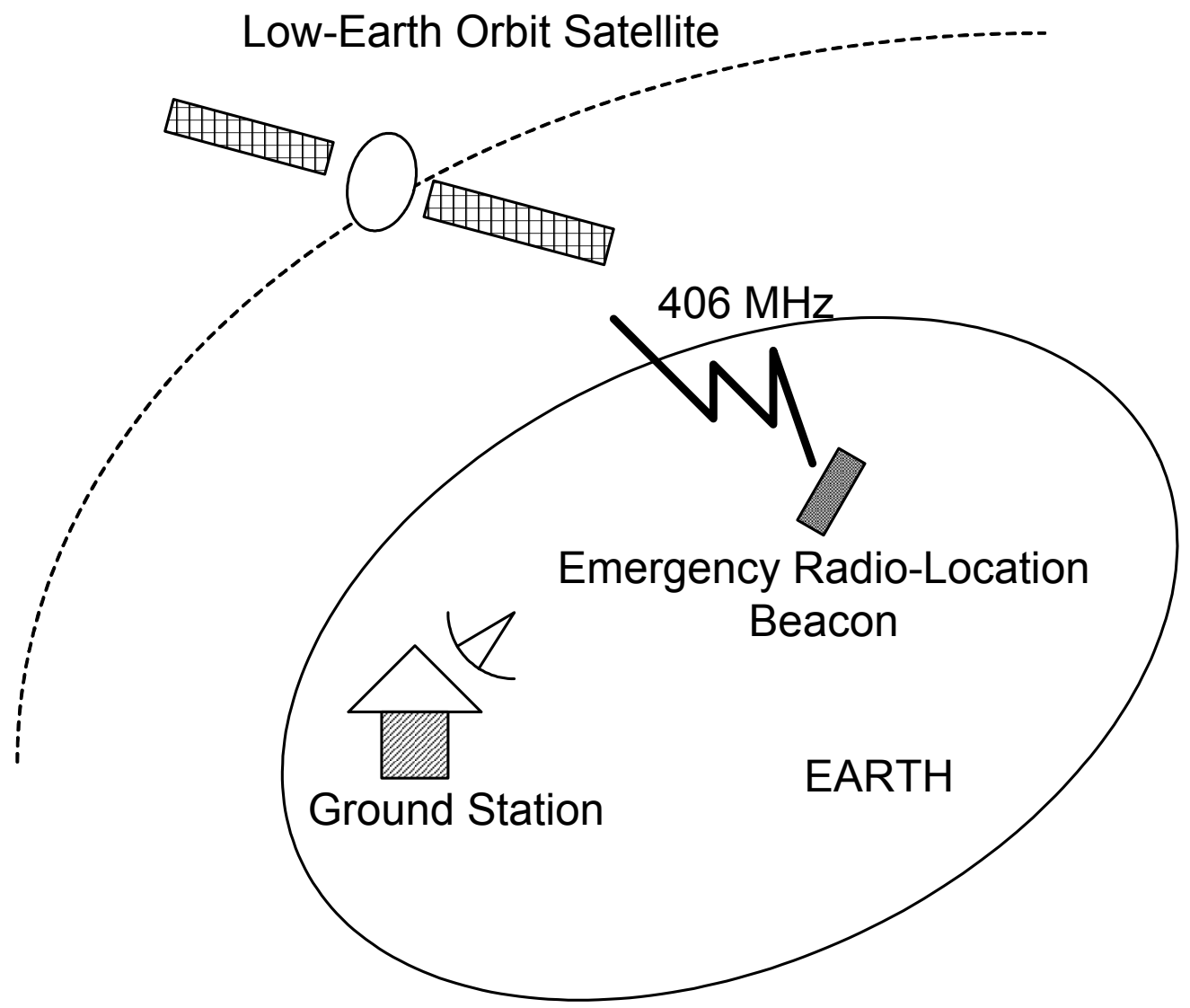

Figure 1.1 SARSAT Emergency Radiolocation Beacon

As a satellite passes over a beacon, the inflection point between the "blue shift" and "red shift" indicates the latitude of the beacon. The slope of the Dopper gradient determines the distance between the satellite and beacon, and with a more accurate measurement to 
account for the rotation of the earth, allows for a determination of the latitude and longitude in a single pass. This leads to a requirement on the rate of frequency drift during changes in ambient temperature of less than $10^{-9}$ per minute [26], which cannot generally be met by temperature-compensated crystal oscillators. The specifications for radiolocation beacons are modest as far as the absolute accuracy of the frequency reference, because the radiolocation system is designed to measure the gradient of the Doppler shift. However, low-power operation is greatly desired to extend the battery life of the distress beacon.

\subsection{High-Accuracy, Low-Power, Low-Cost Frequency Standards}

The preceding examples emphasize the need to develop high-accuracy frequency sources which are also portable and low-cost. The two types of commercially available atomic frequency standards are passive rubidium standards and cesium beam standards. Cesium standards offer the best long-term stability, and accuracy on the order to $10^{-12}$, but are also the most bulky, power hungry, and expensive. Passive rubidium frequency standards are the smallest and lowest-cost atomic standards, and compete with very high-precision oven controlled standards in some applications. In all cases, the challenge is to evaluate the performance of the frequency standard in a variable environment $[27,28]$. For example, if a

crystal oscillator has an acceleration sensitivity of $10^{-8} / \mathrm{g}$, then turning over the crystal unity causes the frequency to change by $10^{-8}$, which may then be the limiting factor for the practical accuracy. Atomic clocks can have very high-accuracy, buy may also have excessive sensitivity to vibrations. Warm-up time is another important problem for both technologies, which prevents greater use of precision frequency control in battery powered systems [30].

The trend in the development of frequency standards is illustrated in Figure 1.2, where although very high-accuracy frequency standards have been established for many years, the push is for portable, robust technologies. 


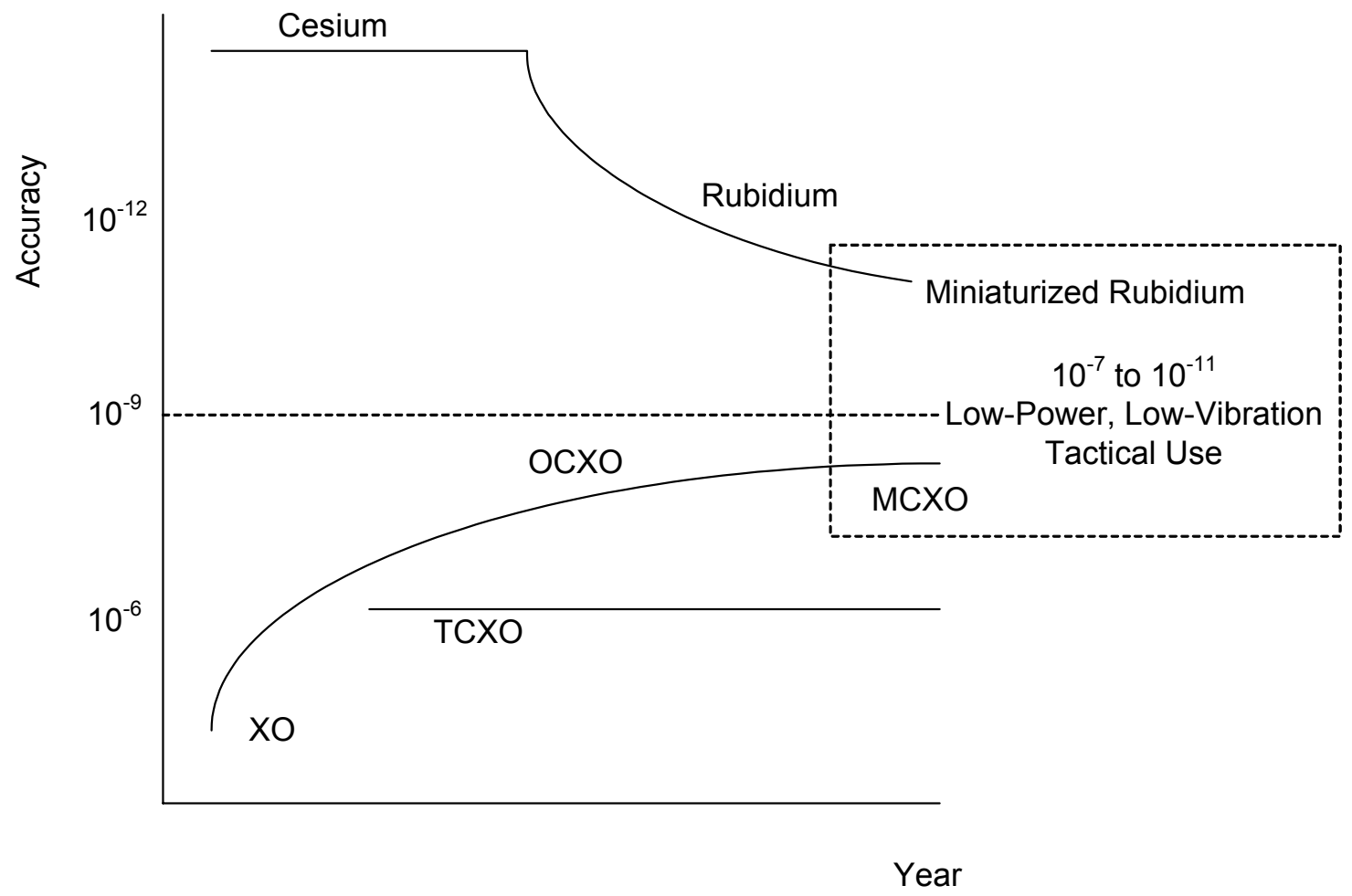

Quartz - has the features of low-power and portability, but not enough accuracy

Classic Atomic Clock - have extremely good stability but not the size, weight, cost, power, robustness for portable use

Figure 1.2: Historical Trends in the Performance of Frequency Standards

Technology developments take the form of improvements in the accuracy of crystal oscillators, and manufacturing innovations to miniaturize passive rubidium standards, with performance converging in the range of $10^{-7}$ to $10^{-10}$ frequency accuracy. Again, the performance evaluation has to account for environmental effects, aging, and vibration, for practical use $[27,28]$. The solid-state atomic frequency standard addresses the need for a robust, low-cost, low-power frequency reference with some of the absolute accuracy and long-term stability characteristics of atomic clocks.

The next section looks more closely at some of the techniques used to optimize the performance of crystal oscillators, and recent work to miniaturize rubidium atomic clocks. 


\subsection{Precision Crystal Oscillators}

In a crystal oscillator, the resonant frequency depends primarily on the precise cut, mass, and dimensions of the crystal resonator. There are various, common techniques for stabilizing frequency over temperature, such as temperature-compensated crystal oscillators (TCXOs), digitally-compensated crystal oscillators (DCXOs), time-compensated clock oscillators (TCCOs), and oven-controlled crystal oscillators (OCXOs). The operating principle, however, is the same in all cases; i.e., the initial frequency is set by the mechanical dimensions of the piezoelectric resonator, and the resonant frequency is stabilized over environmental variations using techniques temperature compensation or temperature regulation.

\section{Power Consumption}

Very good stability over temperature can be achieved for oven-controlled crystal oscillators, in the range of $10^{-9}$ for single oven designs and up to $10^{-10}$ for double oven standards, but power consumption between $0.5 \mathrm{~W}$ and $5 \mathrm{~W}$ is a major shortcoming for portable applications. A microcomputer compensated crystal oscillator (MCXO) uses a look-up table and A/D converter to exactly compensate the temperature vs. frequency characteristics of the crystal resonator. However, hysteresis in the quartz resonator frequency under temperature cycling limits the temperature stability of MCXO's to less than $2 \times 10^{-8}$ [13]. A different technique is direct heating of the quartz crystal for lowpower temperature regulation $[20,21,22]$. A serious shortcoming of this technique results from thermal stress across the crystal resonator, which shifts the resonant frequency.

\section{Aging}

Aging is an inherent characteristic of crystal oscillators [14,15,16,17]. The mechanisms which cause aging include surface contamination of the quartz resonator and stress relief in the crystal mounting. A good quality crystal may exhibit aging in the range of $10^{-9}$ per day, with accuracies in the range of $10^{-6}$ to $10^{-7}$ over one year [1]. For an application which requires an accuracy of $10^{-8}$ and uses an oven-controlled crystal oscillator, there is a 
significant logistics cost for re-calibrating the oscillator [25]. For comparison, a rubidium frequency standard may have aging on the order of $10^{-10}$ per year, which is why atomic clocks must be used to obtain the highest accuracy and long-term stability. However, the low aging rate does not mean than atomic clocks are less sensitivity to vibration than crystal oscillators.

\subsection{Miniaturized Rubidium Atomic Frequency Standards}

Atomic clocks derive a frequency from the quantum mechanical transitions of an ensemble of atoms or ions in the gas phase. Examples range from primary standards such as the cesium beam atomic clock, to smaller clocks such as rubidium standards. Usually, a technique is devised to interrogate the frequency of the magnetic dipole transitions between the hyperfine states of a particular atom or ion vapor, although a standard based on an optical (i.e., electric dipole) transition has been recently implemented [29]. Rubidium standards can be implemented using optical pumping or coherent population trapping to interrogate the hyperfine splitting of the rubidium ions. Regardless of the implementation, the desired frequency is derived from the atomic spectrum of a gas of atoms or ions, rather than from the mechanical dimensions and mass of a piezoelectric resonator. Further details of conventional, miniature atomic clocks may be found in the following references [2-12]. Most recent miniaturized designs use coherent population trapping rather than the traditional optical pumping technique using a rubidium lamp and filter $[6,7,8,9,11]$.

\section{Batch Production}

Producing a reliable, miniaturized atomic frequency standard at reasonable cost requires innovations in manufacturing techniques to fabricate the alkali metal cell and integrate the required optical components. One technique is to etch cavities in borosilicate glass to integrate the lens and vapor cell [4], another combines bulk etching of silicon with bonding to glass $[2,3]$. Alkali metals are highly reactive and therefore designing a stable vapor cell 
is a difficult problem. Other interesting techniques are to fuse hollow-core pyrex fibers [10]. The frequency stability reported for one design is $5 \times 10^{-10}$ at 1 second integration, and an aging rate of $2 \times 10^{-8}$ per day [2].

\subsection{Solid-State Atomic Frequency Standard}

This thesis describes a new class of frequency reference. The frequency source uses the same operating principle as a passive atomic frequency standard; however, the device is entirely solid-state, removing many cost and reliability issues associated with gas-phase atomic clocks. The basis for the solid-state atomic frequency standard is the zero-field electron magnetic resonance of certain transition metal ions doped into dielectric crystals. As Chapters 2 and 3 will describe, the divalent vanadium ion in magnesium oxide has a narrow magnetic resonance spectrum at room temperature. The features of the zero-field magnetic resonance spectra are discussed in detail. Magnesium oxide is a common ceramic, and may be manufactured at very low cost. An important feature of the solid-state atomic frequency standard is that the reference frequency is accuracy defined by the materials system, rather than depending acutely on the manufacturing tolerances as is the case for crystal oscillators.

The apparatus for measuring the "atomic" resonances uses a radio-frequency resonant cavity and a frequency discriminator circuit. The cost of radio-frequency electronics has dropped enormously in the last decade, and particularly for complex radio systems implemented at very low-cost using silicon integrated circuits. The prototype system, described in Chapter 4, uses conventional components, all of which are amenable to implementation in an integrated system, and at room temperature. The heart of the frequency source is an electrical resonator containing a paramagnetic sample. A modulated magnetic field applied to the paramagnetic sample results in frequency modulation of the electrical resonator which the interrogating microwave frequency coincides with the atomic 
resonance. The resulting frequency modulation of the electrical resonator is demodulated using a frequency discriminator circuit, and detected using a lock-in amplifier to measure the atomic resonance.

Chapters 5 and 6 analyze the sensitivity of the magnetic resonance measurement and the stability of passive atomic frequency standards. The results show how the achievable frequency instability is related to the width of the zero-field magnetic resonance and the noise floor of the magnetic resonance measurement. The passive atomic frequency standard is accurately modeled as a linear system, and therefore the analysis of the frequency stability is straightforward. The solid-state atomic clock is presented as a low-cost precision frequency source which, compared to oven-controlled crystal oscillators, may have improved aging, immunity to shock, and power consumption. 


\subsection{Organization}

The organization of the thesis is a follows: The introduction sections gives an overview of the theory of operation and basic design of the solid-state atomic clock, tailored to a general audience. Chapters 2 through 5 treat the theoretical and experimental questions in detail. Chapter 2 introduces the subject of paramagnetic resonance in solids, then develops the specific quantum mechanics which describes the spectrum of the vanadium ion doped in magnesium oxide. In particular, the intensities, frequency, polarization, and Zeeman shift of the zero-field magnetic resonance are calculated, as well as examples of typical spectra. Chapter 3 discusses the resonance widths of dilute paramagnetic solids due to inhomogeneous and homogeneous broadening. We discuss how the magnetic field independent transitions of the low-field spectrum are insensitive to dipolar broadening, and show examples which validate the theory. Chapter 4 is a detailed description of the design and manufacturing of the zero-field magnetic resonance spectrometer which amounts to a proof-of-concept for the solid-state atomic frequency reference. The design and manufacturing of the loop-gap resonator, frequency discriminator, modulation waveforms, and Helmholtz coils are described. Also, heat treatment of vanadium doped magnesium oxide is discussed. Chapter 5 analyzes the noise floor of the spectrometer. Chapter 6 discusses the theoretical and practical frequency stability of the solid-state atomic frequency reference, followed by conclusions and summary of the contributions of the thesis in Chapter 7. 


\section{BIBLIOGRAPHY, CHAPTER 1}

[1] L. L. Lewis, “An introduction to frequency standards," Proceedings of the IEEE, vol. 79, no. 7, pp. 927-935, July 1991.

[2] S. Knappe et al., "A microfabricated atomic clock," Applied Physics Letters, 85, pp. 1460, 2004.

[3] L. Liew et al., "Microfabricated alkali atom vapor cells," Applied Physics Letters, 84, pp. 2694, 2004.

[4] H. C. Nathanson and I. Liberman, United States Patent 6,570,459, "Physics Package Apparatus for an Atomic Clock," Northrop Grumman Corporation, Los Angeles, CA, May 27, 2003.

[5] J. Kitching et al., "Miniature vapor-cell atomic-frequency references," Applied Physics Letters, 81, pp. 553, 2002.

[6] J. Kitching, "Compact atomic clock based on coherent population trapping," Electronics Letters, vol. 37, no. 24, pp. 1449-1451, November 2001.

[7] J. Vanier et al., "The coherent population trapping passive frequency standard [Rb example]," IEEE Trans. on Instrumentation and Measurement, vol. 52, no. 2, pp. 258-262, April 2003.

[8] J. Vanier et al., "Atomic Clocks Based on Coherent Population Trapping: Basic Theoretical Models and Frequency Stability," Proc. IEEE International Frequency Control Symposium, pp. 2-15, 2003.

[9] M. Zhu, "High Contrast Signal in Coherent Population Trapping Based Atomic Frequency Standard Application," Proc. IEEE International Frequency Control Symposium, pp. 16-21, 2003.

[10] S. Knappe et al., "Atomic Vapor Cells for Miniature Frequency References," Proc. IEEE International Frequency Control Symposium, pp. 31-32, 2003.

[11] J. Vanier, "Coherent Population Trapping for the Realization of a Small, Stable, Atomic Clock," Proc. IEEE International Frequency Control Symposium, pp. 424-434, 2002. 
[12] J. Kitching, S. Knappe and L. Holberg, "Performance of Small Scale Frequency References," Proc. IEEE International Frequency Control Symposium, pp. 442-446, 2002.

[13] J. Kusters and J. Vig, "Hysteresis in Quartz Resonators - A Review," IEEE Trans. on Ultrasonics, Ferroelectrics, and Frequency Control, vol. 38, no.3, pp.281-289, May 1991.

[14] F. Walls and J. Vig, "Fundamental Limits on the Frequency Stabilities of Crystal Oscillators," IEEE Trans. on Ultrasonics, Ferroelectrics, and Frequency Control, vol. 42, no. 4, pp. 576-589, July 1995.

[15] R. Filler and J. Vig, "Long-Term Aging in Oscillators," IEEE Trans. on Ultrasonics, Ferroelectrics, and Frequency Control, vol. 40, no. 4, pp.387-394, July 1993.

[16] Y. Yong and J. Vig, "Modeling Resonator Frequency Fluctuations Induced by Absorbing and Desorbing Surface Molecules," IEEE Trans. on Ultrasonics, Ferroelectrics, and Frequency Control, vol. 37, no. 6, pp. 543-550, November 1990.

[17] Y. Yong and J. Vig, "Resonator Surface Contamination - A Cause of Frequency Fluctuations?," IEEE Trans. on Ultrasonics, Ferroelectrics, and Frequency Control, vol. 36, no. 4, pp. 452-458, July 1989.

[18] R. Filler and J. Vig, "Resonators for the Microcomputer Compensated Crystal Oscillators," Proc. 43 ${ }^{\text {rd }}$ Ann. Symposium on Frequency Control, pp. 1-15, 1989.

[19] M. Bloch et al., "Low Power Timekeeping," Proc. 43 ${ }^{\text {rd }}$ Ann. Symposium on Frequency Control, pp. 34-36, 1989.

[20] Garland et al., United States Patent 3,431,392, “Internally Heated Crystal Devices," Hughes Company, Culver City, CA, March 4, 1969.

[21] I. Abramson and A. Dikidzhi, "Improvement of Characteristics of Quartz Resonator-Thermostate with Direct Heating Piezoelement," IEEE Frequency Control Symposium, pp. 499-504, 1992.

[22] I. Abramson, "Internal Heated Quartz Resonator with Low Sensitivity to an Acceleration," IEEE International Frequency Control Symposium, pp. 838-842, 1995.

[23] I. Balaz and M. Minarik, "Towards and OCXO with Infrared Heater," IEEE International Frequency Control Symposium, pp. 674-680, 1996. 
[24] V. E. Bottom, "A History of the Quartz Crystal Industry in the USA," Proc. $35^{\text {th }}$ Ann. Freq. Control. Symposium, pp. 3-12, May 1981.

[25] J. R. Vig, "Military Applications of High Accuracy Frequency Standards and Clocks," IEEE Transactions on Ultrasonics, Ferroelectrics, and Frequency Control, vol. 40, no. 5, pp. 522-527, September 1993.

[26] Specification for COSPAS-SARSAT $406 \mathrm{MHz}$ Distress Beacons, C/S T.001, Issue 3 - Revision 5, October 2003.

[27] IEEE Standard $1193^{\mathrm{TM}}$-2003, IEEE Guide for Measurement of Environmental Sensitivities of Standard Frequency Generators, March 2004.

[28] MIL-PRF-55310D, General Specification for Crystal Controlled Oscillator, March 1998.

[29] S. A. Diddams et al., "An Optical Clock Based on a Single Trapped ${ }^{199} \mathrm{Hg}^{+}$ Ion,” Science, vol. 293, pp. 825-828, August 2001.

[30] T. J. Lynch and J. R. Vaccaro, "Ultrafast Warm-Up Rubidium References," Proc. IEEE International Frequency Control Symposium, pp. 993-1001, 1996. 